\title{
Application of Morphological Operations for Improvement the Segmentation Image of Chicken Intestinal Goblet Cells
}

\author{
Dedi Sepriana \\ Master of Information System \\ Universitas Diponegoro \\ Semarang, Indonesia
}

\author{
Kusworo Adi \\ Department of Physics \\ Universitas Diponegoro \\ Semarang, Indonesia
}

\author{
Catur Edi Widodo \\ Department of Physics \\ Universitas Diponegoro \\ Semarang, Indonesia
}

\begin{abstract}
Goblet Cells identification in the chicken small intestine is generally carried out through a microscope directly. Although identification can be conducted in visual calculation, but in its implementation not only takes a lot of time and effort, but also is relatively subjective. In addition nowadays identification can also be done automatically by digital image processing. This method is in the type of image segmentation. Here the purpose of image segmentation is to separate objects (goblet cells) with other networks. The results of image segmentation are usually still eroded, even objects that stick or merge between several objects, as well as nose around objects and the presence of holes in the object. So that, the morphological operation is needed to improve the image. This study aims to determine the type of morphological operations in improving segmented image results to identify goblet cells of chicken intestine automatically. Erosion, dilation, image filling and open area morphological operations are used to improve segmented image results. In conclusion, we provide that the type of erosion morphology operations, dilation using disk types of 10 pixels, image filling with parameters "holes" and open area of 10 pixels have the greatest percentage of success in improving segmented image results.
\end{abstract}

\section{General Terms}

Dilation, Morphology, Erosion, Image Filling

\section{Keywords}

Goblet Cells Identification, Segmentation, Morphological Surgery.

\section{INTRODUCTION}

Chicken productivity is very influenced by intestinal health. Intestinal health can be seen from the number of goblet cells found in the small intestinal epithelium [1]. Goblet cells have a major role in digestion and absorption of nutrients, and also play an important role as the main producer of mucin [2]. Goblet cells are also an indicator of intestines in a healthy or infected condition [3], the more goblet cells found indicates that intestinal in an unhealthy condition [4]. The number of goblet cells can be calculated in direct by using a microscope by identifying goblet cell object one by one. Although direct visual identification has benefits such as low cost and flexibility, but the procedure takes a lot of time and labor [5]. In addition the subjectivity also influences the process and the results are less precise and accurate [6]. And on its current development, identification can also be obtained automatically by digital image processing. Here the object of goblet cells are separated from other networks through a segmentation process.
The results of image segmentation are usually still eroded, even objects that stick or merge between several objects, as well as nose around objects and the presence of holes in the object. So it is necessary to improve the image of the segmentation results in order to increase the accuracy of identification. The image identification improvement research has been done in several cases. In example of image processing in the segmentation of red blood cells by performing morphological operations in the form of erosion and dilation. Erosion is used to refine the image of separation of cells that accumulate, while dilation is used to corrective in eroded cells image [7]. In addition, the image filling and area operations are used to remove the holes' cells and to mark the edges of cells [8]. From those of experiences we can conclude that we need to use morphological operations in image processing research to improve the image results of segmentation of chicken small intestine goblets. The goblet cells objects that have been separated from other tissues through a segmentation process of two thresholds carried out morphologically. Morphological operations used in this study are erosion, dilation, image filling and open area. Some pixel range is set to determine how many the right morphological operation is to improve segmented image.

\section{LITERATURE REVIEW}

\subsection{Goblet Cells}

Goblet cells are the main producer of mucin, located in the small intestinal epithelium and have a primary role for digestion and absorption of nutrients [2]. Basically, the number of goblet cells will increase due to the food aflatoxin contamination [4]. Several preparation techniques have been determined for counting goblet cells, one of which is AB-PAS staining technique. The AB-PAS staining technique is a dualpurpose method for detecting goblet cells both neutral and acid mucins [9]. The photomicrographs displaying goblet cells with AB-PAS and mucicarmine are shown in Figure 1. The blue object is a goblet cell, while the remain is another networks.

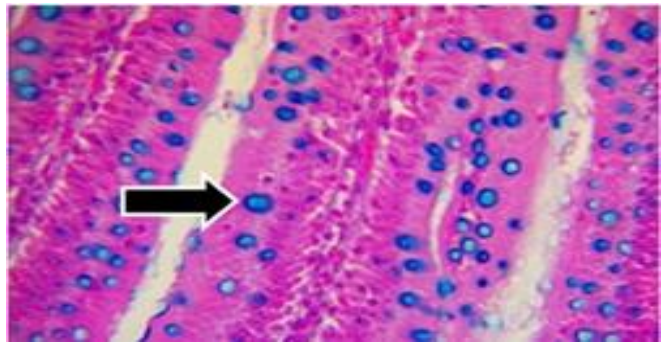

Fig 1: Photomicrograph Showing Goblet Cells with ABPAS Technique [9] 


\subsection{Segmentation}

Blue goblet cell objects can be separated from other tissues by segmentation of two thresholds. Using those two threshold values its implemented is owing to the fact that on the histogram of grayscale images there are peaks that distinguish between one object to another, where the intensity value of object is known to be between $\mathrm{T} 1$ and $\mathrm{T} 2$. The values searching for the construction can be conducted by looking at the gray scale intensity of the image [10]. The following Figure 2 is an example of a gray scale image that has several peaks on the histogram.

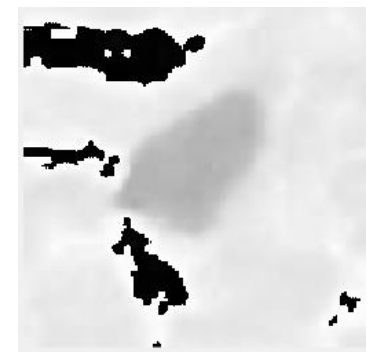

(a) Image of gray scale goblet cell object

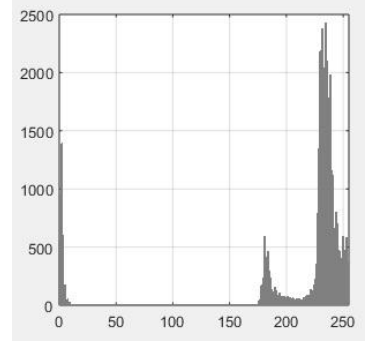

(b) Histogram
Fig 2: The Example of Images with Several Separate Peaks and Valleys

The segmentation formula with two threshold values as follows:

$$
f_{B}(i, j)=\left\{\begin{array}{lc}
1, & T_{1} \leq f_{g}(i, j) \leq T_{2} \\
0, & \text { others }
\end{array}\right.
$$

Where $f_{B}(i, j)$ is a binary image, $f_{g}(i, j)$ is a grayscale image, $\mathrm{T} 1$ is a minimum threshold value and $\mathrm{T} 2$ is a maximum threshold value. The grayscale object with gray scale values less than $\mathrm{T} 1$ and more than $\mathrm{T} 2$ turn into black binary images, while the grayscale object with values equal to or between $\mathrm{T} 1$ and $\mathrm{T} 2$ values change to white binary images.

\subsection{Morphology}

The results of goblet cells segmentation are usually found not similar as the original image, still eroded, even objects that stick or merge between several objects, as well as noise around objects and the presence of holes in the object. In that way, morphological operation is needed to improve the image of the segmentation results. Two types of basic image morphology are dilation and erosion.

Erosion is a morphological operation that will reduce pixels at the boundary between objects in a digital image. Erosion between $\mathrm{A}$ and $\mathrm{B}$ is expressed $\mathrm{A} \ominus \mathrm{B}$ and is defined as follow:

$$
\mathrm{A} \ominus \mathrm{B}=\left\{\mathrm{z} \mid(\mathrm{B})_{\mathrm{z}} \subseteq \mathrm{A}\right)
$$

Erosion A by B is a collection of all points where B is translated by $z$ in content A. B here is structuring element. For grayscale images, the value of the operating result (pixel output) is the minimum value obtained from the set of neighboring pixels. In binary image, if there is a neighboring pixel that has a value of 0 , the pixel output will be set to 0 .

Dilated is a morphological operation that will add pixels to the boundary between objects in a digital image. Dilation between $\mathrm{A}$ and $\mathrm{B}$ is expressed $\mathrm{A} \oplus \mathrm{B}$ and is formulated with following equations.

$\mathrm{A} \oplus \mathrm{B}=\left\{\mathrm{z} \mid(\mathrm{B})_{\mathrm{z}} \cap \mathrm{A} \neq \varnothing\right)$
Dilation A by B is the set of all $\mathrm{Z}$ displacements, as B and A overlap at least one element. The set B is strel, while A is set (the object of the image is distilled). For grayscale images, the value of the operating result (output pixel) is the maximum value obtained from the set of neighboring pixels. In a binary image, if there is a neighboring pixel that has a value of 1 , the pixel output will be set to 1 .

Other morphological operations that can be used in improving segmented image results are the image filling and open area functions. In some segmented images many results show that the presence of holes in objects and noise around objects can reduce the accuracy of identification. To overcome this, you can use the imfill function (BW, "holes") and open area. The imfill function has the function of closing holes in binary imagery [11]. While eliminating noise around the object can utilize the bwareaopen function. Noise around objects that have a pixel area smaller than the specified pixel area will disappear [12].

\section{DESIGN AND IMPLEMENTATION}

Determination of threshold values was calculated by using Matlab Programming Language. It started with determining the object identified as goblet cells from 30 RGB images of object in the small intestine broiler tissue part of the jejunum which had been prepared with AB-PAS staining. After that the darkest blue intensity over the 30 images of intestinal tissue was chosen to be used to find the lower threshold (T1). While the goblet cell object in 30 images of intestinal tissue with the brightest blue intensity was chosen to find the upper threshold (T2).

The selected T1 and T2 threshold is used as a threshold in segmentating goblet cell objects. In the next, the results of segmentation from the previous step are used as objects in image repair morphology operations to obtain the type and range of morphological operations, so that it can be used in image improvement. The improvement of images in the case of increasing the goblet cells identification accuracy includes refining eroded objects even objects that attach or merge between several objects and removing holes at objects and noise around objects. The steps for determining the type and range of morphology are presented in Figure 3.

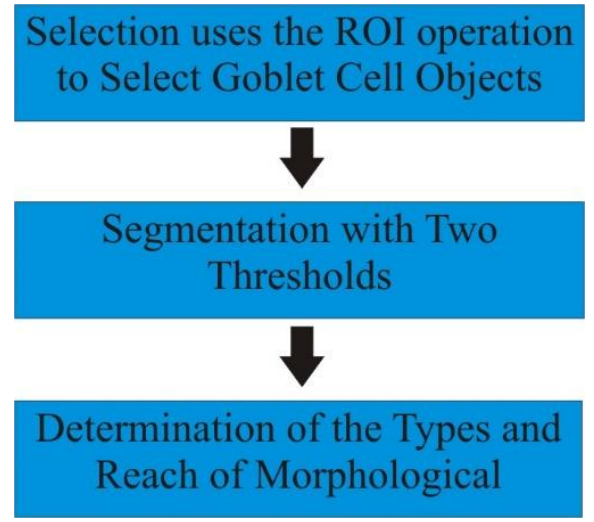

Fig 3: The steps of type and range morphology determination

\subsection{Selection of Goblet Cell Objects}

The goblet cell objects are selected based on the blue colour intensity. The darkest blue color intensity will be used to determine the lower threshold (T1), while the brightest blue colour intensity will be used to determine the upper threshold (T2). The process of determining values of $\mathrm{T} 1$ and $\mathrm{T} 2$ in goblet cell object images begins by selecting the image part 
that its with the darkest and brightest blue intensity by using the ROI (Region Of Interest) process of the square function. The image generated from the ROI process is in the type of a RGB-shaped square image. The selection of goblet cell object by ROI is shown in Figure 4.

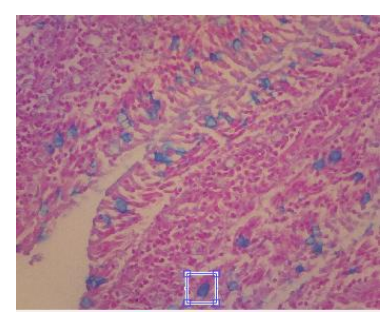

(a) ROI function to select the darkest blue goblet cell object

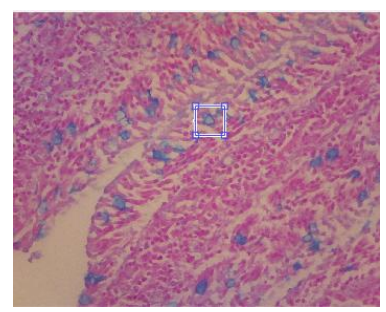

(c) ROI function to select the brightest blue goblet cell object

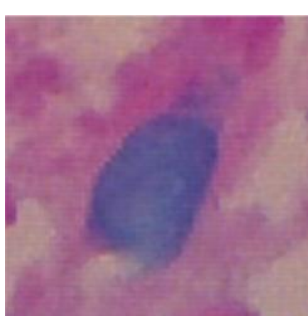

(b) RGB image from ROI selection on the darkest blue goblet cells

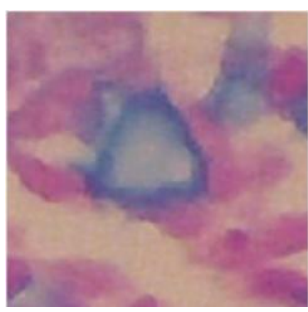

(d) RGB image from ROI selection on the brightest blue goblet cells
Fig 4: Selection of the goblet cell object in the darkest blue and stretched through ROI

The selected image with the ROI process is converted from RGB scale image to HSV. The purpose of this conversion is to select the Hue component as a component used in the process of image conversion to gray scale. Here the Hue component will be extracted from the HSV image. The extraction image result of the Hue component is in a grayfaced image with a double class, so it needs to be converted into uint 8 class.

The image that has been converted into a gray scale is sought for the highest and lowest gray intensity value. Gray scale values in regional pixels are in the range from 0 to 255 . Based on the determining process of the threshold value carried out on 30 images, T1 was obtained with a value of 159 and T2 with a value of 221 . The minimum and maximum gray intensity values obtained will be used in the two threshold values segmentation process. After that, the results obtained from determining the threshold value are tested to determine the identification accuracy of the threshold values.

\subsection{Two Threshold Segmentation}

Segmentation uses two threshold values obtained from the results of determining the threshold value used to separate objects in the form of goblet cells with other networks. Segmentation is carried out on 30 object images used in determining the lower threshold value (T1) and 30 objects used in determining the upper threshold value (T2). Segmentation results obtained 4 groups based on their shape.

The first group of segmentation results is similar to the object. In that case it shows that the results of segmentation with two threshold values can separate the goblet cells object with the background be able to be separated. Beside that, the shape is also almost similar to the goblet cell object on the RGB scale. This result is found in 7 images $\mathrm{T} 1$ determination and 9 images from T2 determination. The example of segmentation result is similar to the original object shown in Figure 5.

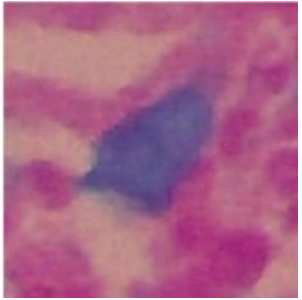

(a) Goblet cell object

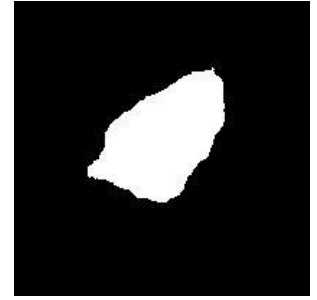

(b) Segmentation result
Fig 5: The segmentation result with background united to object

The second group of segmentation results can separate the goblet cell object with its networks. While there is erosion on several sides of the goblet cell object. This result is found in 8 images from $\mathrm{T} 1$ determination and 10 images from $\mathrm{T} 2$ determination. The example result of segmentation with erosion object in some parts is shown in figure 8 and the examples of segmentation results with results similar to the original object shown in Figure 6.

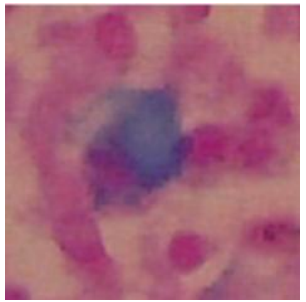

(a) Goblet cell

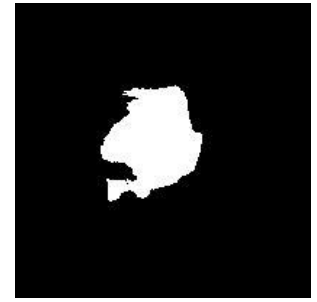

(b) Segmentation result
Fig 6: The segmentation result with eroded object

The third group of segmentation results can separate the goblet cells object with their networks even though there are two adjacent objects and sticking together. This result is found in 3 images of $\mathrm{T} 1$ determination and 2 images $\mathrm{T} 2$ determination. The examples of segmentation results with the results of two adjacent and fused are shown in Figure 7.

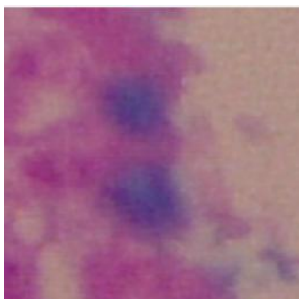

(a) Goblet cell object

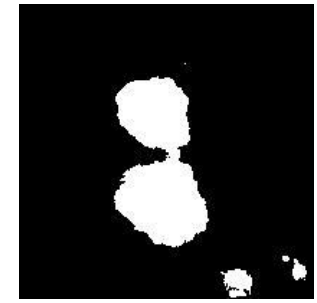

(b) Segmentation result
Fig 7: The segmentation result with two adjacent and fused objects

The fourth group of segmentation result can separate the object in the form of a goblet cell with a margin with a noise around the object and hole in the object. This result is found in 12 images from the determination of $\mathrm{T} 1$ and 9 images from the determination of $\mathrm{T} 2$. The example of segmentation with result similar to the original object shown in figure 8 . 


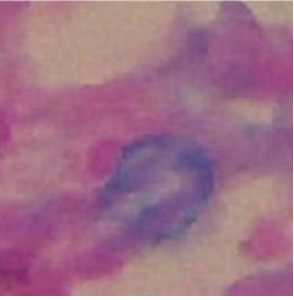

(a) Goblet cell object

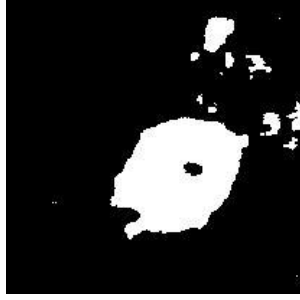

(b) Segmentation result

Fig 8: The segmentation result with noise around objects and holes in the object

Ihe result indicates that the determination of two threshold values T1 and T2 with the values 159 and 221 respectively can separate the goblet cells object with other networks. However, there is still some imperfect object separation from the background, but this is still in tolerable and can be improved by using morphological operations [13].

In the first group of segmentation, the object is expected to be more similar to the original object if the morphology operation process is implemented. The second group with morphological operations is expected to be able to refine eroded objects. The third group of morphological operations is expected can be separated. While the fourth group with morphological operations is expected to be able to remove holes in the object and eliminate noise around the object.

\subsection{Determination of the Type and Range of Morphological Operations}

The determination of type and range will decide the success of image improvement. Morphological operations used in this study are in the type of erosion, dilation, image filling and open area. The erosion morphology operations and dilation are using disk types with a range of 7,10 and 14 pixels, image filling with the parameter "holes" and open areas with a range of 7, 10 and 14 pixels. The morphological operations are implemented on the four groups of objects resulting from the previous determination of the threshold values by 60 images.

\section{RESULTS}

In the first group of segmentation with two threshold values can separate the goblet cells object with the background. Moreover from the process of morphological operations determination here, the result of object segmentation is almost similar to the original object and shown in Figure 9.

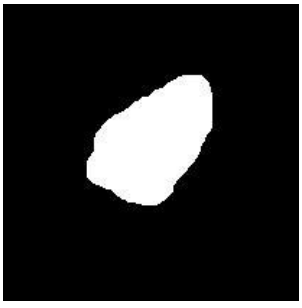

(a) Segmented object result

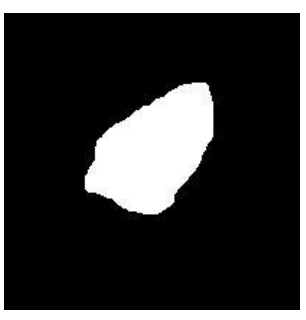

(b) The morphological operation with the parameter of "holes" and range of 7 pixels

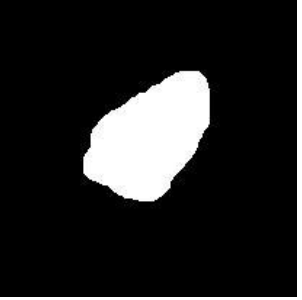

(c) The morphological operation with the parameter of "holes" and range of 10 pixels

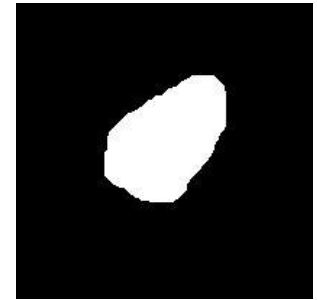

(d) The morphological operation with the parameter of "holes" and range of 14 pixels

Fig 9: The morphological operations in the result of object segmentation are almost similar to the original object

Here the detail operations result in the first group is shown in the Table 1 with the 7 images of $\mathrm{T} 1$ and 9 images of T2.

Table 1. Table results of morphological operations in the result of flash segmentation with the original object

\begin{tabular}{|c|c|c|c|c|c|c|}
\hline \multirow{2}{*}{ Object } & \multicolumn{2}{|c|}{ 7 Pixels } & \multicolumn{2}{c|}{ 10 Pixels } & \multicolumn{2}{c|}{ 14 Pixels } \\
\cline { 2 - 7 } & Succeed & Failed & Succeed & Failed & Succeed & Failed \\
\hline T1 & 6 & 1 & 6 & 1 & 6 & 1 \\
\hline T2 & 8 & 1 & 9 & 0 & 6 & 3 \\
\hline Total & 14 & 2 & 15 & 1 & 12 & 4 \\
\hline Percentag & $88 \%$ & & $94 \%$ & & $75 \%$ & \\
\hline
\end{tabular}

Here the detail operations result in the second group is shown in the Table 2 with the 8 images of $\mathrm{T} 1$ and 10 images of T2.

Table 2. Table results of morphological operations in eroded object

\begin{tabular}{|c|c|c|c|c|c|c|}
\hline \multirow{2}{*}{ Object } & \multicolumn{2}{|c|}{7 Pixels } & \multicolumn{2}{c|}{10 Pixels } & \multicolumn{2}{c|}{14 Pixels } \\
\cline { 2 - 7 } & Succeed & Failed & Succeed & Failed & Succeed & Failed \\
\hline $\mathrm{T} 1$ & 5 & 3 & 8 & 0 & 5 & 3 \\
\hline $\mathrm{T} 2$ & 7 & 3 & 9 & 1 & 5 & 5 \\
\hline Total & 12 & 6 & 17 & 1 & 10 & 8 \\
\hline Percentage & $67 \%$ & & $94 \%$ & & $56 \%$ & \\
\hline
\end{tabular}

In the second group of object segmentation, there is erosion on several sides of the goblet cell object and the morphological operations is expected to be able to refine eroded objects. Determination of morphological operations in the second group is shown in Figure 10.

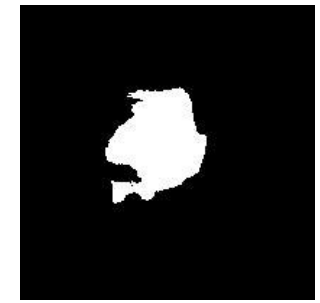

(a) Segmented object result

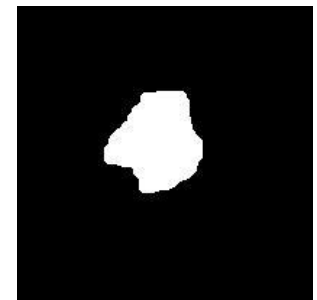

(b) The morphological operation with the parameter of "holes" and range of 7 pixels 


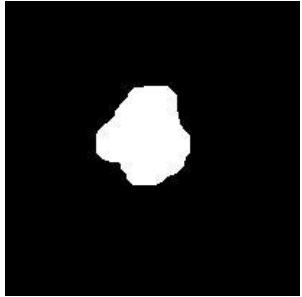
operation with the parameter of "holes" and range of 10 pixels (c) The morphological

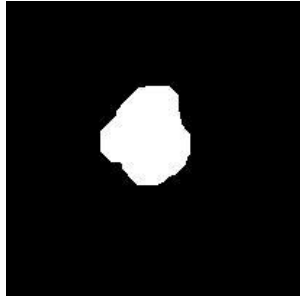

(d) The morphological of "holes" and range of 14 pixels operation with the parameter

Fig 10: The morphological operations in the eroded object In the third group of segmentation, there are two adjacent objects and sticking together, and morphological operations here is expected be able to separate the objects. The determination of morphological operations in the third group of object segmentation results with several unified objects is shown in Figure 11

Here the detail operations result in the third group is shown in the Table 3 with the 3 images of $\mathrm{T} 1$ and 2 images of $\mathrm{T} 2$.

Table 3. Table results of morphological operations in the several objects merge

\begin{tabular}{|c|c|c|c|c|c|c|}
\hline \multirow{2}{*}{ Object } & \multicolumn{2}{|c|}{7 Pixels } & \multicolumn{2}{c|}{10 Pixels } & \multicolumn{2}{c|}{14 Pixels } \\
\cline { 2 - 7 } & Succeed & Failed & Succeed & Failed & Succeed & Failed \\
\hline T1 & 2 & 1 & 2 & 1 & 2 & 1 \\
\hline T2 & 2 & 0 & 2 & 0 & 1 & 1 \\
\hline Total & 4 & 1 & 4 & 1 & 3 & 2 \\
\hline Percentage & $80 \%$ & & $80 \%$ & & $60 \%$ & \\
\hline
\end{tabular}

The fourth group of segmentation result can separate the object but still leave it a noise and hole. Here the morphological operations is expected to be able to remove holes and eliminate noise around the object. Determination of morphological operations in the fourth group is shown in Figure 12. In addition the detail operations result in the fourth group is shown in the Table 4 with the 12 images of $\mathrm{T} 1$ and 9 images of $\mathrm{T} 2$.

Table 4. Table results of morphological operations in the object with hole and noise

\begin{tabular}{|c|c|c|c|c|c|c|}
\hline \multirow{2}{*}{ Object } & \multicolumn{2}{|c|}{7 Pixels } & \multicolumn{2}{c|}{ 10 Pixels } & \multicolumn{2}{c|}{14 Pixels } \\
\cline { 2 - 7 } & Succeed & Failed & Succeed & Failed & Succeed & Failed \\
\hline $\mathrm{T} 1$ & 9 & 3 & 11 & 1 & 11 & 1 \\
\hline $\mathrm{T} 2$ & 4 & 5 & 6 & 3 & 3 & 6 \\
\hline Total & 13 & 8 & 17 & 4 & 14 & 7 \\
\hline Percentage & $62 \%$ & & $81 \%$ & & $67 \%$ & \\
\hline
\end{tabular}

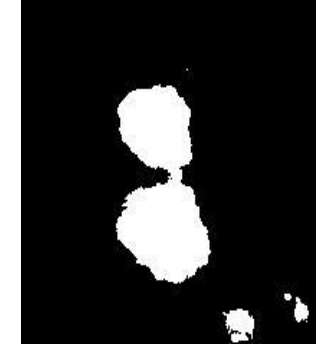

(a) segmented object result

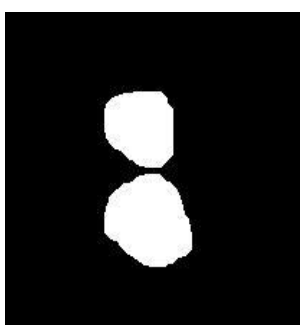

(c) The morphological operation with the parameter of "holes" and range of 10 pixels

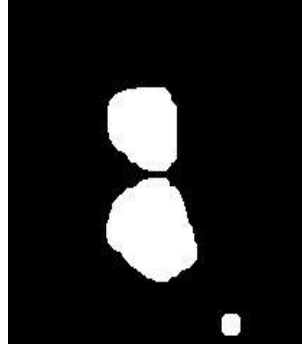

(b) The morphological operation with the parameter of "holes" and range of 7 pixels

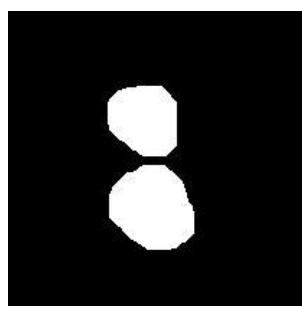

(d) The morphological operation with the parameter of "holes" and range of 14 pixels
Fig 11: The morphological operations in the several objects merge

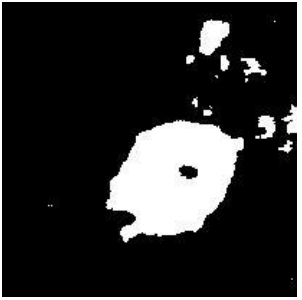

(a) Segmented result

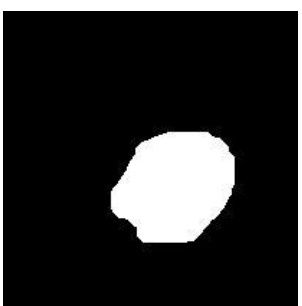

(c) The morphological operation with the parameter of "holes" and range of 10 pixels

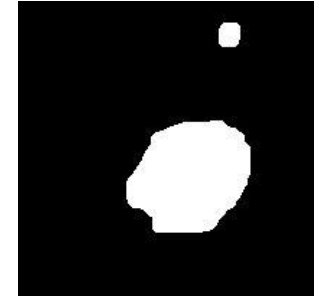

(b) The morphological operation with the parameter of "holes" and range of 7 pixels

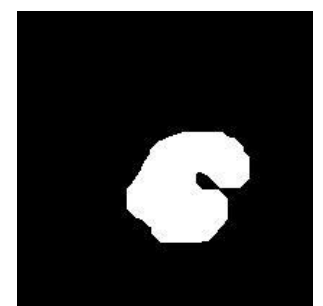

(d) The morphological operation with the parameter of "holes" and range of 14 pixels
Fig 12: The morphological operations in object with holes and noise around 


\section{CONCLUSION}

The determination of morphological operations which performed on the segmented objects with 30 images of $\mathrm{T} 1$ and 30 images of T2 as presented in the Table 1, Table 2, Table 3 and Table 4 obtained the results as follows:

1. Erosion morphology operations with 7 pixel disk type dilation, 7 pixel open area and image filling with "holes" parameters show the average success of improving the image by $74 \%$.

2. Erosion morphology operations with dilation of 10 pixel disk type, open 10 pixel area and image filling with parameter "holes" show the average success of improving the image by $87 \%$.

3. Erosion morphological operations with dilation 14 pixel disk type, 14 pixel open area and image filling with "holes" parameters show the average success of improving the image by $64 \%$.

Based on the average success obtained in the morphology operation, it can be concluded that erosion morphology operations with 10 pixel disk type dilation, 10 pixel open area and image filling with "holes" parameter has the highest success rate of improving the image that is equal to $87 \%$. So with that result, its morphological operation is highly recomended in improving segmented image to increase the accuracy of goblet cells identification.

\section{ACKNOWLEDGMENTS}

This study was supported by the Pathology and Anatomy laboratory of the Faculty of Medicine, Vocational School Laboratory of the Animal Health of Gajah Mada University and Animal Health Laboratory of the Semarang Veterinary Center. We are grateful to Mr. Yunadir, Mrs. Drh. F. Sri Wiryaningsih and Mr. Drh. Hendra for help in these experiments, deliver the valuable discussions, and delighted to explain the characteristics and analysis of this work.

\section{REFERENCES}

[1] Horn, N. L., Donkin, S. S., Applegate, T. J., and Adeola, O. 2009. Intestinal mucin dynamics: response of broiler chicks and White Pekin ducklings to dietary threonine. Poultry science. 88(9), 1906-1914.

[2] Contreras-Ruiz, L., Ghosh-Mitra, A., Shatos, M. A., Dartt, D. A., and Masli, S. 2013. Modulation of conjunctival goblet cell function by inflammatory cytokines. Mediators of inflammation.
[3] Johnston, G. 2010. Automated handheld instrument improves counting precision across multiple cell lines. BioTechniques. 48(4), 325-327.

[4] Jahanian, E., Mahdavi, A. H., Asgary, S., and Jahanian, R. 2016. Effect of dietary supplementation of mannanoligosaccharides on growth performance, ileal microbial counts, and jejunal morphology in broiler chicks exposed to aflatoxins. Livestock Science. 190, 123-130.

[5] Kim, S. I., Kim, H. J., Lee, H. J., Lee, K., Hong, D., Lim, H., and Yi, Y. W. 2016. Application of a non-hazardous vital dye for cell counting with automated cell counters. Analytical biochemistry. 492, 8-12.

[6] Ji, X., Zhao, C., Gong, P., Li, Q., and Yu, Y. 2016 Research on high-accuracy biological microscopic imaging and cell counting system. Optik-International Journal for Light and Electron Optics. 127(23), 11483 11491

[7] Sharif, J. M., Miswan, M. F., Ngadi, M. A., Salam, M. S. H., and bin Abdul Jamil, M. M. 2012. Red blood cell segmentation using masking and watershed algorithm: A preliminary study. In International Conference on Biomedical Engineering (ICoBE). 258-262.

[8] Santos, E. M. D. S., and Marcal, A. R. S. 2017. Segmentation of microscopic images for pollen grain detection. In 8th International Conference of Pattern Recognition Systems. 1-6.

[9] Osho, S. O., Wang, T., Horn, N. L., and Adeola, O. 2017. Comparison of goblet cell staining methods in jejunal mucosa of turkey poults. Poultry science. 96(3), 556-559.

[10] Smith, A. R. 1978. Color gamut transform pairs. ACM Siggraph Computer Graphics. 12(3), 12-19.

[11] Bhat, R., and Mehandia, B. 2014. Recognition of vehicle number plate using matlab. International journal of innovative research in electrical, electronics, instrumentation and control engineering. 2(8), 18991903.

[12] Viazzi, S., Bahr, C., Van Hertem, T., Schlageter-Tello, A., Romanini, C. E. B., Halachmi, I., and Berckmans, D. 2014. Comparison of a three-dimensional and twodimensional camera system for automated measurement of back posture in dairy cows. Computers and Electronics in Agriculture. 100, 139-147. 